This item was submitted to Loughborough's Research Repository by the author.

Items in Figshare are protected by copyright, with all rights reserved, unless otherwise indicated.

\title{
Augmented interface systems for the Darcy-Stokes problem
}

PLEASE CITE THE PUBLISHED VERSION

http://dx.doi.org/10.1007/978-3-642-35275-1_48

\section{PUBLISHER}

(c) Springer-Verlag Berlin Heidelberg

\section{VERSION}

AM (Accepted Manuscript)

\section{PUBLISHER STATEMENT}

This work is made available according to the conditions of the Creative Commons Attribution-NonCommercialNoDerivatives 4.0 International (CC BY-NC-ND 4.0) licence. Full details of this licence are available at: https://creativecommons.org/licenses/by-nc-nd/4.0/

\section{LICENCE}

CC BY-NC-ND 4.0

\section{REPOSITORY RECORD}

Discacciati, Marco. 2019. “Augmented Interface Systems for the Darcy-stokes Problem”. figshare. https://hdl.handle.net/2134/18750. 


\title{
Augmented interface systems for the Darcy-Stokes problem
}

\author{
Marco Discacciati \\ Laboratori de Càlcul Numèric (LaCàN), Universitat Politècnica de Catalunya (UPC \\ BarcelonaTech), Campus Nord UPC - C2, E-08034 Barcelona, Spain. \\ marco.discacciatieupc.edu
}

Summary. In this paper we study interface equations associated to the Darcy-Stokes problem using the classical Steklov-Poincaré approach and a new one called augmented. We compare these two families of methods and characterize at the discrete level suitable preconditioners with additive and multiplicative structures. Finally, we present some numerical results to assess their behavior in presence of small physical parameters.

\section{Introduction and problem setting}

Let $\Omega \subset \mathbb{R}^{d}$ ( $\left.d=2,3\right)$ be a bounded domain decomposed into two non intersecting subdomains: $\Omega_{f}$, filled by a viscous incompressible fluid, and $\Omega_{p}$, formed by a porous medium, separated by an interface $\Gamma=\bar{\Omega}_{f} \cap \bar{\Omega}_{p}$. The fluid in $\Omega_{f}$ has no free surface and it can filtrate through the adjacent porous medium. The motion of the fluid in $\Omega_{f}$ is described by the Stokes equations:

$$
-v \triangle \mathbf{u}+\nabla p=\mathbf{f}, \quad \operatorname{div} \mathbf{u}=0 \quad \text { in } \Omega_{f}
$$

where $v>0$ is the kinematic viscosity, while $\mathbf{u}$ and $p$ are the velocity and pressure. In $\Omega_{p}$ we describe the fluid motion by the equations:

$$
\mathbf{u}_{p}=-\mathrm{K} \nabla \varphi, \quad \operatorname{div} \mathbf{u}_{p}=0 \quad \text { in } \Omega_{p}
$$

where $\mathbf{u}_{p}$ is the fluid velocity, $\varphi$ the piezometric head and $\mathrm{K}$ the hydraulic conductivity tensor. The first equation is Darcy's law that provides the simplest linear relation between velocity and pressure in porous media. We can equivalently rewrite (2) as the elliptic equation involving only the piezometric head:

$$
-\operatorname{div}(\mathrm{K} \nabla \varphi)=0 \quad \text { in } \Omega_{p}
$$

Besides suitable boundary conditions on $\partial \Omega$, we supplement the Darcy-Stokes problem (1), (3) with the following coupling conditions on $\Gamma$ :

$$
-\mathrm{K} \nabla \varphi \cdot \mathbf{n}=\mathbf{u} \cdot \mathbf{n}, \quad-\mathbf{n} \cdot \mathrm{T}(\mathbf{u}, p) \cdot \mathbf{n}=g \varphi, \quad-\varepsilon \tau \cdot \mathrm{T}(\mathbf{u}, p) \cdot \mathbf{n}=v \mathbf{u} \cdot \boldsymbol{\tau},
$$

where $\mathrm{T}(\mathbf{u}, p)$ is the fluid stress tensor, $\tau$ denotes a set of linear independent unit tangential vectors to $\Gamma$ and $\varepsilon$ is a coefficient related to the characteristic length of the pores of the porous medium. Conditions (4) $)_{1}$ and $(4)_{2}$ impose the continuity of the normal velocity and of the normal component of the normal stress on $\Gamma$. The so-called Beavers-Joseph-Saffman condition 
$(4)_{3}$ does not yield any coupling but provides a boundary condition for the Stokes problem since it involves only quantities in the domain $\Omega_{f}$. For more details we refer to $[9,11,12,14]$.

\section{Interface equations associated to the Darcy-Stokes problem}

In $[7,8]$, we showed that the coupled Darcy-Stokes problem can be reformulated in terms of the solution of equations defined only on the interface $\Gamma$ involving suitable Steklov-Poincaré operators associated to the subproblems in $\Omega_{f}$ and $\Omega_{p}$. We formally briefly review this approach referring to the cited works for more details.

If we select as interface variable $\lambda \in H_{00}^{1 / 2}(\Gamma)$ to represent the normal velocity across $\Gamma$ : $\lambda=\mathbf{u} \cdot \mathbf{n}=-\mathrm{K} \nabla \varphi \cdot \mathbf{n}$ on $\Gamma$, we can express the solution of the Darcy-Stokes problem in terms of the solution of the interface equation: find $\lambda \in H_{00}^{1 / 2}(\Gamma)$ such that

$$
\left\langle S_{s} \lambda, \mu\right\rangle+\left\langle S_{d} \lambda, \mu\right\rangle=\left\langle\chi_{s}, \mu\right\rangle+\left\langle\chi_{d}, \mu\right\rangle \quad \forall \mu \in H_{00}^{1 / 2}(\Gamma) .
$$

Equation (5) imposes the continuity condition (4) $)_{2}$. The linear continuous operators $\chi_{s}$ and $\chi_{d}$ depend on the data of the problem and $\langle\cdot, \cdot\rangle$ denotes the duality pairing between $H_{00}^{1 / 2}(\Gamma)$ and its dual $\left(H_{00}^{1 / 2}(\Gamma)\right)^{\prime}$. Concerning $S_{s}$ and $S_{d}$, we remark that

- the operator $S_{s}: H_{00}^{1 / 2}(\Gamma) \rightarrow\left(H_{00}^{1 / 2}(\Gamma)\right)^{\prime}$ maps the space of normal velocities on $\Gamma$ to the space of normal stresses on $\Gamma$ through the solution of a Stokes problem in $\Omega_{f}$ with boundary condition $\mathbf{u} \cdot \mathbf{n}=\lambda$ on $\Gamma$.

- $S_{d}$ maps the space of fluxes of $\varphi$ on $\Gamma$ to the space of traces of $\varphi$ on $\Gamma$ via the solution of a Darcy problem in $\Omega_{p}$ with the boundary condition $-\mathrm{K} \nabla \varphi \cdot \mathbf{n}=\lambda$ on $\Gamma$. The operator $S_{d}$ should be a map between $H^{-1 / 2}(\Gamma)$ and $H^{1 / 2}(\Gamma)$, but in (5) we are applying it to $H_{00}^{1 / 2}(\Gamma)$, a space with a higher regularity than needed where we cannot guarantee the coercivity of the operator.

On the other hand, if we choose as interface unknown $\eta \in H^{1 / 2}(\Gamma)$ the trace of the piezometric head on $\Gamma: \eta=g \varphi_{\mid \Gamma}=-\mathbf{n} \cdot \mathrm{T}(\mathbf{u}, p) \cdot \mathbf{n}$ on $\Gamma$, the Darcy-Stokes problem can be equivalently reformulated as find $\eta \in H^{1 / 2}(\Gamma)$ :

$$
\left\langle S_{f} \eta, \mu\right\rangle+\left\langle\left\langle S_{p} \eta, \mu\right\rangle=\left\langle\left\langle\chi_{f}, \mu\right\rangle+\left\langle\left\langle\chi_{p}, \mu\right\rangle \quad \forall \mu \in H^{1 / 2}(\Gamma),\right.\right.\right.
$$

where $\chi_{f}$ and $\chi_{p}$ are linear continuous operators depending on the data of the problem. Equation (6) imposes the coupling condition (4) 1 . Here:

- the operator $S_{f}$ maps the space of normal stresses on $\Gamma$ to the space of normal velocities on $\Gamma$ via the solution of a Stokes problem with the boundary condition $-\mathbf{n} \cdot \mathbf{T}(\mathbf{u}, p) \cdot \mathbf{n}=\eta$ on $\Gamma$. This operator would naturally be defined from $H^{-1 / 2}(\Gamma)$ to $H_{00}^{1 / 2}(\Gamma)$ so that in (6) we are applying it to functions with a higher regularity than needed.

- The operator $S_{p}: H^{1 / 2}(\Gamma) \rightarrow\left(H^{1 / 2}(\Gamma)\right)^{\prime}$ maps the space of traces of $\varphi$ on $\Gamma$ to the space of fluxes of $\varphi$ on $\Gamma$ by solving a Darcy problem in $\Omega_{p}$ with the Dirichlet boundary condition $g \varphi=\eta$ on $\Gamma$. 


\section{Augmented interface equations}

The classical approach summarized in Sect. 2 leads to reformulate the Darcy-Stokes problem as interface equations depending on a single interface unknown: either $\lambda$, the normal velocity across $\Gamma$, or $\eta$, the piezometric head on $\Gamma$. We have remarked that the Steklov-Poincaré operators $S_{d}$ and $S_{f}$ are not acting on their natural functional spaces, but they are assigned functions with higher regularity than expected. This prevents us from guaranteeing their coerciveness (see [7]). In this section we present a different approach based on [5, 3, 4, 6] consisting in writing the coupled Darcy-Stokes problem as a system of linear equations on $\Gamma$ involving both variables $\lambda$ and $\eta$.

\subsection{The augmented Dirichlet-Dirichlet problem}

To obtain the augmented Dirichlet-Dirichlet (aDD) formulation assume that $\lambda \in H_{00}^{1 / 2}(\Gamma)$ is equal to the normal velocity $\mathbf{u} \cdot \mathbf{n}$ on $\Gamma$, but not necessarily to the conormal derivative of $\varphi$ on $\Gamma$. On the other hand, let $\eta \in H^{1 / 2}(\Gamma)$ be equal to the trace of $\varphi$ on $\Gamma$ but not to the normal component of the Cauchy stress of the Stokes problem on $\Gamma$. Then, to recover the solution of the original Darcy-Stokes problem we have to impose both the continuity of normal velocity and of normal stresses:

$$
\begin{array}{ll}
-\int_{\Gamma} \mathbf{n} \cdot \mathrm{T}(\mathbf{u}(\lambda), p(\lambda)) \cdot \mathbf{n} \mu=\int_{\Gamma} \eta \mu & \forall \mu \in H_{00}^{1 / 2}(\Gamma) \\
-\int_{\Gamma} \mathrm{K} \nabla \varphi(\eta) \cdot \mathbf{n} \xi=\int_{\Gamma} \lambda \xi & \forall \xi \in H^{1 / 2}(\Gamma) .
\end{array}
$$

Using the definition of the Steklov-Poincaré operators, we can rewrite these conditions as: find $(\lambda, \eta) \in H_{00}^{1 / 2}(\Gamma) \times H^{1 / 2}(\Gamma)$ such that

$$
\begin{array}{ll}
\left\langle S_{s} \lambda, \mu\right\rangle+\langle\eta, \mu\rangle=\left\langle\chi_{s}, \mu\right\rangle & \forall \mu \in H_{00}^{1 / 2}(\Gamma) \\
\left\langle\left\langle S_{p} \eta, \xi\right\rangle-\langle\lambda, \xi\rangle=\left\langle\left\langle\chi_{p}, \xi\right\rangle\right.\right. & \forall \xi \in H^{1 / 2}(\Gamma),
\end{array}
$$

or, in operator form:

$$
\left(\begin{array}{cc}
S_{s} & \mathscr{I} \\
-\mathscr{J} & S_{p}
\end{array}\right)\left(\begin{array}{l}
\lambda \\
\eta
\end{array}\right)=\left(\begin{array}{l}
\chi_{s} \\
\chi_{p}
\end{array}\right)
$$

where $\mathscr{I}: H^{1 / 2}(\Gamma) \rightarrow\left(H_{00}^{1 / 2}(\Gamma)\right)^{\prime}$ and $\mathscr{J}: H_{00}^{1 / 2}(\Gamma) \rightarrow\left(H^{1 / 2}(\Gamma)\right)^{\prime}$ are linear continuous maps.

We call (8) augmented Dirichlet-Dirichlet (aDD) formulation because both functions $\lambda$ and $\eta$ play the role of Dirichlet boundary conditions for the Stokes and the Darcy subproblems, respectively. Notice that we are imposing the equalities (8) in the sense of dual spaces and that the operators $S_{s}$ and $S_{p}$ still act on their natural functional spaces.

\subsection{The augmented Neumann-Neumann problem}

We follow now a similar approach to Sect. 3.1, but we assume that $\lambda \in H^{-1 / 2}(\Gamma)$ is equal to the conormal derivative of the piezometric head $-\mathrm{K} \nabla \varphi \cdot \mathbf{n}$ on $\Gamma$ and $\eta \in H^{-1 / 2}(\Gamma)$ is equal to the normal component of the fluid Cauchy stress on $\Gamma$. Then, to recover the solution of the original problem we impose the following equalities:

$$
\begin{array}{ll}
\int_{\Gamma} \mathbf{u}(\eta) \cdot \mathbf{n} \mu=\int_{\Gamma} \lambda \mu & \forall \mu \in H^{-1 / 2}(\Gamma) \\
\int_{\Gamma} \varphi(\lambda) \xi=-\int_{\Gamma} \eta \xi & \forall \xi \in H^{-1 / 2}(\Gamma) .
\end{array}
$$

Using the definition of the Steklov-Poincaré operators, we can rewrite these conditions as: find $(\lambda, \eta) \in H^{-1 / 2}(\Gamma) \times H^{-1 / 2}(\Gamma)$ such that 


$$
\begin{array}{ll}
\left\langle S_{f} \eta, \mu\right\rangle_{*}-\langle\lambda, \mu\rangle_{*}=\left\langle\chi_{f}, \mu\right\rangle_{*} & \forall \mu \in H^{-1 / 2}(\Gamma) \\
\left\langle\left\langle S_{d} \lambda, \xi\right\rangle_{*}+\langle\eta \eta, \xi\rangle_{*}=\left\langle\left\langle\chi_{d}, \xi\right\rangle_{*}\right.\right. & \forall \xi \in H^{-1 / 2}(\Gamma),
\end{array}
$$

corresponding to the operator form:

$$
\left(\begin{array}{cc}
S_{d} & \mathscr{I}_{*} \\
-\mathscr{J}_{*} & S_{f}
\end{array}\right)\left(\begin{array}{l}
\lambda \\
\eta
\end{array}\right)=\left(\begin{array}{l}
\chi_{d} \\
\chi_{f}
\end{array}\right) .
$$

Here $\mathscr{I}_{*}: H^{-1 / 2}(\Gamma) \rightarrow H^{1 / 2}(\Gamma)$ and $\mathscr{J}_{*}: H^{-1 / 2}(\Gamma) \rightarrow H_{00}^{1 / 2}(\Gamma)$ are linear continuous maps, while $\langle\cdot, \cdot\rangle_{*}$ and $\langle\cdot, \cdot\rangle_{*}$ denote the corresponding pairing.

We call this formulation augmented Neumann-Neumann (aNN) because both functions $\lambda$ and $\eta$ play the role of Neumann boundary conditions for the Darcy and the Stokes subproblems, respectively.

The aNN formulation may be regarded as the "dual" of the aDD approach. Notice that the operators $S_{f}$ and $S_{d}$ are now acting on their natural spaces, differently form the classical setting of Sect. 2. The analysis of problems (8) and (10) can be carried out following the guidelines of [5].

\section{Algebraic formulation of the interface problems}

We consider a finite element discretization of the coupled problem using conforming grids across the interface $\Gamma$. The discrete spaces for the Stokes problem satisfy the inf-sup condition. In this way we obtain the linear system:

$$
\left(\begin{array}{cccc}
F & D & 0 & 0 \\
D^{T} & A_{\Gamma \Gamma} & 0 & -M_{\Gamma} \\
0 & 0 & C_{i i} & C_{i \Gamma} \\
0 & M_{\Gamma}^{T} & C_{\Gamma i} & C_{\Gamma \Gamma}
\end{array}\right)\left(\begin{array}{c}
\boldsymbol{u}_{i} \\
\boldsymbol{u}_{\Gamma} \\
\boldsymbol{\varphi}_{i} \\
\boldsymbol{\varphi}_{\Gamma}
\end{array}\right)=\left(\begin{array}{c}
\boldsymbol{f}_{f i} \\
\boldsymbol{f}_{f \Gamma} \\
\boldsymbol{f}_{p i} \\
\boldsymbol{f}_{p \Gamma}
\end{array}\right)
$$

where $\boldsymbol{u}_{\Gamma}$ is the vector of the nodal values of the normal velocity on $\Gamma$ while $\boldsymbol{u}_{i}$ is the vector of the remaining degrees of freedom (velocity and pressure) in $\Omega_{f}$. On the other hand, $\varphi_{\Gamma}$ is the vector of the (unknown) values of $\varphi$ on $\Gamma$ while $\boldsymbol{\varphi}_{i}$ corresponds to the remaining degrees of freedom in $\Omega_{p}$.

The discrete counterpart of the Steklov-Poincaré operators can be found computing the Schur complement systems corresponding to either $\boldsymbol{u}_{\Gamma}$ or $\boldsymbol{\varphi}_{\Gamma}$. Precisely, we find:

$$
\begin{array}{ll}
\Sigma_{s}=A_{\Gamma \Gamma}-D^{T} F^{-1} D, & \Sigma_{f}=M_{\Gamma}^{T} \Sigma_{s}^{-1} M_{\Gamma}, \\
\Sigma_{p}=C_{\Gamma \Gamma}-C_{\Gamma i} C_{i i}^{-1} C_{i \Gamma}, & \Sigma_{d}=M_{\Gamma} \Sigma_{p}^{-1} M_{\Gamma}^{T} .
\end{array}
$$

The characterization of these discrete operators in terms of the associated Darcy or Stokes problems in $\Omega_{p}$ and $\Omega_{f}$ allows us to provide upper and lower bounds for their eigenvalues. Assuming $v$ and $\mathrm{K}$ constants in $\Omega_{f}$ and $\Omega_{p}$, respectively, and the computational mesh to be uniform and regular, we can find (see $[7,13,15]$ ) ( $\preceq$ indicates that the inequalities hold up to constants independent of $h, v, \mathrm{~K})$ :

$$
\begin{array}{ll}
h v \preceq \sigma\left(\Sigma_{s}\right) \preceq v, & h^{2} v^{-1} \preceq \sigma\left(\Sigma_{f}\right) \preceq h v^{-1} \\
h \mathrm{~K} \preceq \sigma\left(\Sigma_{p}\right) \preceq \mathrm{K}, & h^{2} \mathrm{~K}^{-1} \preceq \sigma\left(\Sigma_{d}\right) \preceq h \mathrm{~K}^{-1}
\end{array}
$$

The discrete counterparts of the interface problems (5), (6), (8), (10) read:

- Discrete interface equation for the normal velocity: find $\boldsymbol{u}_{\Gamma}$ such that

$$
\Sigma_{s} \boldsymbol{u}_{\Gamma}+\Sigma_{d} \boldsymbol{u}_{\Gamma}=\boldsymbol{\chi}_{s}+\boldsymbol{\chi}_{d} .
$$


- Discrete interface equation for the piezometric head: find $\varphi_{\Gamma}$ such that

$$
\Sigma_{f} \boldsymbol{\varphi}_{\Gamma}+\Sigma_{p} \boldsymbol{\varphi}_{\Gamma}=\boldsymbol{\chi}_{f}+\boldsymbol{\chi}_{p}
$$

- Discrete aDD problem: find $\left(\boldsymbol{u}_{\Gamma}, \boldsymbol{\varphi}_{\Gamma}\right)$ such that

$$
\left(\begin{array}{cc}
\Sigma_{s} & -M_{\Gamma} \\
M_{\Gamma}^{T} & \Sigma_{p}
\end{array}\right)\left(\begin{array}{l}
\boldsymbol{u}_{\Gamma} \\
\boldsymbol{\varphi}_{\Gamma}
\end{array}\right)=\left(\begin{array}{l}
\boldsymbol{\chi}_{s} \\
\boldsymbol{\chi}_{p}
\end{array}\right)
$$

- Discrete aNN problem: find $\left(\boldsymbol{u}_{\Gamma}, \boldsymbol{\varphi}_{\Gamma}\right)$ such that

$$
\left(\begin{array}{cc}
\Sigma_{d} & M_{\Gamma} \\
-M_{\Gamma}^{T} & \Sigma_{f}
\end{array}\right)\left(\begin{array}{l}
\boldsymbol{u}_{\Gamma} \\
\boldsymbol{\varphi}_{\Gamma}
\end{array}\right)=\left(\begin{array}{l}
\boldsymbol{\chi}_{d} \\
\boldsymbol{\chi}_{f}
\end{array}\right)
$$

The augmented approach allows to compute both interface variable at once but it requires to solve a system whose dimension is twice the one of the classical methods.

\section{Iterative solution methods and numerical results}

We present now some numerical methods to solve problems (14)-(17) focusing on cases where the fluid viscosity $v$ and the hydraulic conductivity $\mathrm{K}$ are small. These are indeed situations of interest for most practical applications. In [10] a Robin-Robin method was proposed to solve effectively (14). Here we adopt the generalized Hermitian/skew-Hermitian splitting (GHSS) method of [2] for (14) and (15) and the HSS method of [1] for (16) and (17). We start considering (14).

The matrix $\Sigma_{s}+\Sigma_{d}$ has no skew-symmetric component being symmetric positive definite, but thanks to the estimates (13) we can mimick the splitting proposed in [2] considering $\Sigma_{S}$ as a matrix multiplied by a coefficient $(v)$ which may become small. Thus, we can characterize the preconditioner for (14):

$$
P_{1}=\left(2 \alpha_{1}\right)^{-1}\left(\Sigma_{s}+\alpha_{1} I\right)\left(\Sigma_{d}+\alpha_{1} I\right) .
$$

Proceeding analogously for (15), we can characterize the preconditioner

$$
P_{2}=\left(2 \alpha_{2}\right)^{-1}\left(\Sigma_{p}+\alpha_{2} I\right)\left(\Sigma_{f}+\alpha_{2} I\right) .
$$

Preconditioners $P_{1}$ and $P_{2}$ involve suitable acceleration parameters $\alpha_{1}$ and $\alpha_{2}$ and can be used within GMRES iterations. Remark that they can be regarded as generalizations of the Robin-Robin method introduced in [7, 10].

On the other hand, as the matrices in (16) and (17) are positive skew-symmetric with symmetric positive definite diagonal blocks, we apply the HSS splitting proposed in [1] separating the symmetric and the skew-symmetric parts of the matrices. Thus, we can characterize the following preconditioners for GMRES iterations for (16) and (17), respectively, with $\alpha_{3}, \alpha_{4}$ suitable acceleration parameters:

$$
\begin{gathered}
P_{3}=\left(2 \alpha_{3}\right)^{-1}\left(\begin{array}{cc}
\Sigma_{s}+\alpha_{3} I & 0 \\
0 & \Sigma_{p}+\alpha_{3} I
\end{array}\right)\left(\begin{array}{cc}
\alpha_{3} I & -M_{\Gamma} \\
M_{\Gamma}^{T} & \alpha_{3} I
\end{array}\right) \\
P_{4}=\left(2 \alpha_{4}\right)^{-1}\left(\begin{array}{cc}
\Sigma_{d}+\alpha_{4} I & 0 \\
0 & \Sigma_{f}+\alpha_{4} I
\end{array}\right)\left(\begin{array}{cc}
\alpha_{4} I & M_{\Gamma} \\
-M_{\Gamma}^{T} & \alpha_{4} I
\end{array}\right) .
\end{gathered}
$$

According to [2] these preconditioners are effective when either the skew-symmetric or the symmetric part dominates. Thanks to (13) we can expect that for small $v$ and $\mathrm{K}$ the skewsymmetric part dominates in (16) and the symmetric one in (17). 
All preconditioners $P_{i}$ require the solution of a Stokes problem in $\Omega_{f}$ and of a Darcy problem in $\Omega_{p}$. However, $P_{1}$ and $P_{2}$ have a multiplicative structure while in $P_{3}$ and $P_{4}$ the two subproblems may be solved in a parallel fashion. They are all effective when $v$ and $\mathrm{K}$ become small. A thorough study of these preconditioners will make the object of a future work, where also the choice of the parameters $\alpha_{i}$ will be analyzed. For the tests reported in table 1, following [2], we set $\alpha_{1}, \alpha_{3} \simeq \sqrt{v}, \alpha_{2} \simeq \sqrt{\mathrm{K}}$ and $\alpha_{4} \simeq 10^{-1}$. However, a better characterization of such parameters is necessary to have a more robust behavior of the preconditioners, independent of both the mesh size and of the coefficients $v$ and $\mathrm{K}$.

In the numerical tests, both the Stokes and the Darcy subproblems are solved via direct methods. The matrices in (20) and (21) involving $M_{\Gamma}$ and $I$ are assembled explicitly and the associated linear systems are solved using direct methods. We consider $\Omega_{f}=(0,1) \times(1,2)$, $\Omega_{p}=(0,1)^{2}$ with interface $\Gamma=(0,1) \times\{1\}$ and the analytic solution: $\mathbf{u}=\left((y-1)^{2}+(y-1)+\right.$ $1, x(x-1)), p=2 v(x+y-1), \varphi=\mathrm{K}^{-1}\left(x(1-x)(y-1)+(y-1)^{3} / 3\right)+2 v x$. A comparison with preconditioners $\Sigma_{s}$ for (14) and $\Sigma_{p}$ for (15) studied in [7] is also presented. Although such preconditioners are optimal with unitary $v$ and $\mathrm{K}$, they perform quite poorly when small viscosities and permeabilities are considered.

\section{References}

[1] Z.-Z. Bai, G.H. Golub, and M.K. Ng. Hermitian and skew-Hermitian splitting methods for non-Hermitian positive definite linear systems. SIAM J. Matrix Anal. Appl., 24(3): 603-626, 2003.

[2] M. Benzi. A generalization of the Hermitian and skew-Hermitian splitting iteration. SIAM J. Matrix Anal. Appl., 31(2):360-374, 2009.

[3] P.J. Blanco, R.A. Feijóo, and S.A. Urquiza. A unified variational approach for coupling 3D-1D models and its blood flow applications. Comput. Methods Appl. Mech. Engrg., 196(41-44):4391-4410, 2007.

[4] P.J. Blanco, R.A. Feijóo, and S.A. Urquiza. A variational approach for coupling kinematically incompatible structural models. Comput. Methods Appl. Mech. Engrg., 197 (17-18):1577-1602, 2008.

[5] P.J. Blanco, M. Discacciati, and A. Quarteroni. Modeling dimensionally-heterogeneous problems: analysis, approximation and applications. Numer. Math., 119(2):299-335, 2011.

[6] P.J. Blanco, P. Gervasio, and A. Quarteroni. Extended variational formulation for heterogeneous partial differential equations. Computational Methods in Applied Mathematics, 11(2):141-172, 2011.

[7] M. Discacciati. Domain Decomposition Methods for the Coupling of Surface and Groundwater Flows. PhD thesis, EPFL, 2004.

[8] M. Discacciati and A. Quarteroni. Analysis of a domain decomposition method for the coupling of Stokes and Darcy equations. In F. Brezzi et al., editor, Numerical Mathematics and Advanced Applications, ENUMATH 2001, pages 3-20. Springer. Milan, 2003.

[9] M. Discacciati and A. Quarteroni. Navier-Stokes/Darcy coupling: modeling, analysis, and numerical approximation. Rev. Mat. Com., 22:315-426, 2009.

[10] M. Discacciati, A. Quarteroni, and A. Valli. Robin-Robin domain decomposition methods for the Stokes-Darcy coupling. SIAM J. Numer. Anal., 45(3):1246-1268, 2007.

[11] W. Jäger and A. Mikelić. On the boundary conditions at the contact interface between a porous medium and a free fluid. Ann. Scuola Norm. Sup. Pisa Cl. Sci., 23:403-465, 1996. 
Table 1. Number of iterations to solve (14)-(17) using different preconditioners. Four computational meshes $\left(h_{j}=2^{-(j+1)}\right)$ and several values of $v$ and $\mathrm{K}$ have been considered.

GMRES iterations without and with preconditioner for $(14)\left(\right.$ tol $\left.=10^{-7}\right)$.

\begin{tabular}{cccccccccc} 
& \multicolumn{3}{c}{$v=10^{-4}, \mathrm{~K}=10^{-3}$} & \multicolumn{3}{c}{$v=10^{-6}, \mathrm{~K}=10^{-5}$} & \multicolumn{3}{c}{$v=10^{-6}, \mathrm{~K}=10^{-8}$} \\
\cline { 2 - 10 } & No prec. & $\Sigma_{s}$ & $P_{1}$ & No prec. & $\Sigma_{s}$ & $P_{1}$ & No prec. & $\Sigma_{s}$ & $P_{1}$ \\
\hline$h_{1}$ & 8 & 8 & $4\left(\alpha_{1}=10^{-2}\right)$ & 8 & 8 & $3\left(\alpha_{1}=10^{-3}\right)$ & 8 & 8 & $3\left(\alpha_{1}=10^{-3}\right)$ \\
$h_{2}$ & 16 & 15 & $5\left(\alpha_{1}=10^{-2}\right)$ & 16 & 15 & $3\left(\alpha_{1}=10^{-3}\right)$ & 16 & 15 & $3\left(\alpha_{1}=10^{-3}\right)$ \\
$h_{3}$ & 26 & 20 & $7\left(\alpha_{1}=10^{-3}\right)$ & 26 & 20 & $3\left(\alpha_{1}=10^{-3}\right)$ & 26 & 20 & $3\left(\alpha_{1}=10^{-3}\right)$ \\
$h_{4}$ & 33 & 17 & $7\left(\alpha_{1}=10^{-3}\right)$ & 33 & 17 & $4\left(\alpha_{1}=10^{-3}\right)$ & 33 & 17 & $3\left(\alpha_{1}=10^{-3}\right)$ \\
\hline
\end{tabular}

GMRES iterations without and with preconditioner for $(15)\left(\mathrm{tol}=10^{-7}\right)$.

\begin{tabular}{cccccccccc} 
& \multicolumn{3}{c}{$v=10^{-4}, \mathrm{~K}=10^{-3}$} & \multicolumn{3}{c}{$v=10^{-6}, \mathrm{~K}=10^{-5}$} & \multicolumn{3}{c}{$v=10^{-6}, \mathrm{~K}=10^{-8}$} \\
\cline { 2 - 10 } & No prec. & $\Sigma_{p}$ & $P_{2}$ & No prec. & $\Sigma_{p}$ & $P_{2}$ & No prec. & $\Sigma_{p}$ & $P_{2}$ \\
\hline$h_{1}$ & 9 & 9 & $6\left(\alpha_{2}=10^{-2}\right)$ & 9 & 9 & $4\left(\alpha_{2}=10^{-3}\right)$ & - & - & $3\left(\alpha_{2}=10^{-3}\right)$ \\
$h_{2}$ & 17 & 17 & $7\left(\alpha_{2}=10^{-2}\right)$ & 17 & 17 & $4\left(\alpha_{2}=10^{-3}\right)$ & - & - & $3\left(\alpha_{2}=10^{-3}\right)$ \\
$h_{3}$ & 32 & 31 & $8\left(\alpha_{2}=10^{-2}\right)$ & 33 & 33 & $5\left(\alpha_{2}=10^{-3}\right)$ & 33 & 33 & $4\left(\alpha_{2}=10^{-3}\right)$ \\
$h_{4}$ & 46 & 42 & $8\left(\alpha_{2}=10^{-2}\right)$ & 59 & 57 & $5\left(\alpha_{2}=10^{-3}\right)$ & 63 & 62 & $4\left(\alpha_{2}=10^{-3}\right)$ \\
\hline
\end{tabular}

GMRES iterations without and with preconditioner $P_{3}$ for $(16)\left(\right.$ tol $\left.=10^{-9}\right)$.

\begin{tabular}{ccccccc} 
& \multicolumn{2}{c}{$v=10^{-4}, \mathrm{~K}=10^{-3}$} & \multicolumn{2}{c}{$v=10^{-6}, \mathrm{~K}=10^{-5}$} & \multicolumn{2}{c}{$v=10^{-6}, \mathrm{~K}=10^{-8}$} \\
\cline { 2 - 7 } & No prec. & \multicolumn{1}{c}{$P_{3}$} & No prec. & $P_{3}$ & No prec. & $P_{3}$ \\
\hline$h_{1}$ & 17 & $14\left(\alpha_{3}=10^{-3}\right)$ & 17 & $7\left(\alpha_{3}=10^{-3}\right)$ & 17 & $8\left(\alpha_{3}=10^{-3}\right)$ \\
$h_{2}$ & 33 & $17\left(\alpha_{3}=10^{-3}\right)$ & 33 & $8\left(\alpha_{3}=10^{-3}\right)$ & 33 & $10\left(\alpha_{3}=10^{-3}\right)$ \\
$h_{3}$ & 63 & $22\left(\alpha_{3}=5 \cdot 10^{-4}\right)$ & 65 & $8\left(\alpha_{3}=5 \cdot 10^{-4}\right)$ & 65 & $10\left(\alpha_{3}=5 \cdot 10^{-4}\right)$ \\
$h_{4}$ & 67 & $23\left(\alpha_{3}=5 \cdot 10^{-4}\right)$ & 79 & $9\left(\alpha_{3}=5 \cdot 10^{-4}\right)$ & 101 & $11\left(\alpha_{3}=5 \cdot 10^{-4}\right)$ \\
\hline
\end{tabular}

GMRES iterations without and with preconditioner $P_{4}$ for $(17)\left(\right.$ tol $\left.=10^{-9}\right)$.

\begin{tabular}{ccccccc} 
& \multicolumn{2}{c}{$v=10^{-4}, \mathrm{~K}=10^{-3}$} & \multicolumn{2}{c}{$v=10^{-6}, \mathrm{~K}=10^{-5}$} & \multicolumn{2}{c}{$v=10^{-6}, \mathrm{~K}=10^{-8}$} \\
\cline { 2 - 8 } & No prec. & \multicolumn{1}{c}{$P_{4}$} & No prec. & $P_{4}$ & No prec. & $P_{4}$ \\
\hline$h_{1}$ & 17 & $16\left(\alpha_{4}=0.1\right)$ & 16 & $9\left(\alpha_{4}=0.5\right)$ & 9 & $8\left(\alpha_{4}=1\right)$ \\
$h_{2}$ & 32 & $18\left(\alpha_{4}=0.1\right)$ & 32 & $8\left(\alpha_{4}=0.5\right)$ & 16 & $7\left(\alpha_{4}=0.5\right)$ \\
$h_{3}$ & 59 & $20\left(\alpha_{4}=5 \cdot 10^{-2}\right)$ & 58 & $10\left(\alpha_{4}=0.1\right)$ & 30 & $5\left(\alpha_{4}=0.8\right)$ \\
$h_{4}$ & 82 & $27\left(\alpha_{4}=5 \cdot 10^{-2}\right)$ & 81 & $8\left(\alpha_{4}=0.1\right)$ & 44 & $5\left(\alpha_{4}=0.8\right)$ \\
\hline
\end{tabular}

[12] W. Jäger and A. Mikelić. On the interface boundary condition of Beavers, Joseph and Saffman. SIAM J. Appl. Math., 60:1111-1127, 2000.

[13] L. Lakatos. Numerical analysis of iterative substructuring methods for the Stokes/Darcy problem. Master's thesis, EPFL, 2010.

[14] W.L. Layton, F. Schieweck, and I. Yotov. Coupling fluid flow with porous media flow. SIAM J. Num. Anal., 40:2195-2218, 2003.

[15] A. Quarteroni and A. Valli. Domain Decomposition Methods for Partial Differential Equations. Oxford University Press, New York, 1999. 